\title{
Inibição do Receptor Plaquetário Glicoproteína (GP) IIb/IIIa em Síndromes Isquêmicas Agudas e Intervenções Coronárias
}

\author{
Paulo R. A. Caramori, José R. Casco, Alcides J. Zago, Allan G. Adelman
}

Toronto, Canadá - Porto Alegre, RS

\begin{abstract}
A progressiva compreensão do papel primordial que as plaquetas desempenham na doença cardiovascular, particularmente em síndromes isquêmicas agudas, tem aberto grandes oportunidades terapêuticas em cardiologia ${ }^{1-4}$. Os bloqueadores do receptor plaquetário glicoproteína (GP) IIb/IIIa, que inibem a via comum da agregação plaquetária, possuem um grande potencial terapêutico em pacientes submetidos a intervenções coronárias e no manejo de síndromes coronárias agudas. $\mathrm{O}$ anticorpo monoclonal contra a GP IIb/IIIa, ou abciximab, tem sido extensivamente estudado em pacientes submetidos a intervenções coronárias $^{5-11}$, sendo o primeiro de uma série de agentes a ser introduzido para o uso clínico. Um número expressivo de outros bloqueadores peptídicos e não peptídicos do receptor plaquetário GP IIb/IIIa também estão sendo avaliados em estudos clínicos de fase II e III ${ }^{12-19}$. Esta atualização tem por finalidade descrever como estes potentes agentes antiplaquetários atuam e discutir as situações clínicas em que há benefício potencial ou demonstrado com o seu uso, baseado nos resultados de estudos clínicos randomizados. Adicionalmente, discutiremos o uso dos bloqueadores do receptor GP IIb/ IIIa em associação com stents em intervenções coronárias.
\end{abstract}

\section{Síndromes isquêmicas agudas e os receptores GP IIb/IIIa}

A ruptura do endotélio sobre uma placa arterosclerótica é responsável pela maioria das síndromes coronárias agudas. A exposição dos componentes subendoteliais inicia a cascata da coagulação e provê as condições para ativação plaquetária ${ }^{20}$. As plaquetas possuem receptores que interagem com glicoproteínas subendoteliais, como o colágeno e o fator von Willebrand, aderindo ao tecido vascular desprovido de endotélio. A adesão estimula a ativação plaquetária. As plaquetas ativadas sofrem modificações conformacionais com aumento da sua superfície e liberam agonistas como o tromboxano $\mathrm{A}_{2}$, a adenosina difosfa-

Universidade de Toronto e Hospital de Clínicas de Porto Alegre - UFRS. The Cardiovascular Clinical Research Laboratory, Mount Sinai Hospital. Correspondência: Dr. Paulo Caramori - Hospital de Clínicas de Porto Alegre. Serviço de Cardiologia. Rua Ramiro Barcelos 2350. Porto Alegre, RS, Brasil. 90035 003. E-mail: Caramori.P@plug-in.com.br

Recebido para publicação em 20/2/98

Aceito em 23/4/98 to e a serotonina ${ }^{21}$. Estes agonistas, por sua vez, ativam outras plaquetas. As alterações conformacionais secundárias a ativação levam à exteriorização dos receptores GP IIb/ IIIa na superfície da membrana plaquetária ${ }^{22-24}$. Os receptores GP IIb/IIIa ligam-se a proteínas plasmáticas, como o fibrinogênio e o fator von Willebrand, determinando a agregação plaquetária ${ }^{25}$.

A cascata da coagulação ativada leva a conversão do fibrinogênio à fibrina. A fibrina estabiliza as pontes que ligam as plaquetas, criando uma rede de plaquetas e fibrina. Paralelamente, a cascata da coagulação forma trombina, que é um potente ativador plaquetário, e amplifica o processo de agregação ${ }^{26}$. A trombina liga-se a hemácias e ao agregado plaquetário, formando um coágulo rico em plaquetas. Isto causa obstrução total ou parcial do fluxo sangüíneo através da artéria coronária acometida, causando isquemia miocárdica ${ }^{27,28}$.

O receptores GP IIb/IIIa são os receptores mais abundantes na superfície plaquetária ${ }^{29}$. Eles pertencem a uma grande família de receptores, comumente denominados, "integrinas" 30,31, que são responsáveis pelas interações célula-célula e célula-proteínas em diversos processos biológicos. Estes receptores são moléculas heterodiméricas, constituídos de duas cadeias de proteínas transmembrana, as subunidades $\alpha$ e $\beta$. O receptor GP IIb/IIIa é constituído pela subunidade $\alpha_{\mathrm{IIb}}$ e pela subunidade $\beta_{3}{ }^{32,33}$. A associação $\mathrm{GP}$ IIb/IIIa $\left(\alpha \operatorname{IIb} \beta_{3}\right)$ parece ocorrer apenas em plaquetas. Contudo, a subunidade GPIIIa $\left(\beta_{3}\right)$ forma complexos com uma outra subunidade $\alpha$, a subunidade $\alpha$, para formar o complexo $\alpha_{v} \beta_{3}$. O receptor GP $\alpha_{v} \beta_{3}$ interage com a vitronectina ${ }^{34}$,estando presente nas células endoteliais, células musculares lisas vasculares e, em pequeno número, nas plaquetas ${ }^{34,35}$.

Oreceptor GP IIb/IIIIa reconhece dois sítios específicos na molécula de fibrinogênio. O primeiro é a seqüência aminoácida RGD (arginina-glicina-ácido aspártico) que é encontrada no fibrinogênio, no fator von Willebrand e em outras proteínas como a vitronectina e a fibronectina ${ }^{36-38}$. O fibrinogênio difere da outras proteínas de adesão por ter duas seqüências RGD por cada meia molécula ${ }^{39}$. O segundo sitio é a sequiência de aminoácidos KQAGDV (glicinaglutamina-alanina-glicina-ácido aspártico-valina) que ocorre apenas no fibrinogênio, sendo o principal sítio de ligação do fibrinogênio no receptor GP IIb/IIIa ${ }^{40}$. Devido a esses múltiplos sítios de ligação, o fibrinogênio tem uma afinidade muito grande pelos receptores GP IIb/IIIa ${ }^{41}$. 
O tratamento padrão das síndromes isquêmicas agudas e da oclusão trombótica, durante ou após uma angioplastia, inclui a administração do ácido-acetilsalicílico como agente antiplaquetário ${ }^{42-45}$. O ácido-acetilsalicílico acetila de maneira irreversível a ciclo-oxigenase plaquetária e inibe a produção de tromboxano $\mathrm{A}_{2}{ }^{46}$. Contudo, o ácido-acetilsalicílico é um inibidor plaquetário relativamente fraco ${ }^{46,47}$, bloqueando, aproximadamente $20 \%$ da agregação plaquetária. O ácido-acetilsalicílico não possui efeito sobre a trombina e possui pouco ou nenhum efeito sobre outros potentes ativadores plaquetários, como a adenosina difosfato, a vasopressina, a serotonina, o colágeno, o fibrinogênio e a fibronectina ${ }^{48}$. Além disso, o ácido-acetilsalicílico promove a produção de leucotrienos, que causam vasoespasmo e podem aumentar a adesividade plaquetária ${ }^{49}$. O ácidoacetilsalicílico também bloqueia a produção de prostaciclina endotelial, que atuaria como um vasodilatador e promotor da fibrinólise ${ }^{50}$. Em adição, há uma significativa variação individual nos efeitos do ácido-acetilsalicílico. Aproximadamente, $40 \%$ dos pacientes são pelo menos, parcialmente, resistente às suas propriedades antiplaquetárias ${ }^{51}$. Apesar destas limitações, o ácido-acetilsalicílico permanece como a terapia antiplaquetária mais comumente usada.

\section{Bloqueadores dos receptores GP IIb/IIIa plaquetários}

A descoberta dos receptores plaquetários GP IIb/IIIa por Barry Coller ${ }^{52}$, há mais de uma década, levou ao desenvolvimento de agentes antiplaquetários direcionados contra este receptor ${ }^{53}$. Vários agentes peptídicos e não peptídicos têm sido produzidos, baseado na seqüência de aminoácidos RGD, crucial na ligação de várias substâncias a esses receptores. Estes antagonistas do receptor GP IIb/IIIa incluem o eptifibatide, que é um heptapeptídio cíclico (Integrilin, Cor Therapeutics, São Francisco, EUA) ${ }^{54}$, o lamifibam, que é um pseudo-peptídio (Hoffman-LaRoche, Basel, Suiça) ${ }^{55}$, e o tirofibam, que é um derivado não peptídico da tirosina (Merck, West Point, EUA) ${ }^{56}$. Agentes ativos oralmente, como o xemilofibam (Searle, Skokie, EUA) ${ }^{57,58}$, também têm sido desenvolvidos. Esses agentes estão sendo submetidos a estudos clínicos fase II e III para a prevenção de complicações trombótica em síndromes coronárias isquêmicas e em revascularização percutânea. Contudo, o inibidor dos receptores GP IIb/IIIa que será discutido em maior detalhe nesse artigoé o anticorpo monoclonal c7E3 Fab ou abciximab $\left(\right.$ ReoPro $\left.^{\circledR}\right)$, que se encontra disponível para uso clínico.

O abciximab é o bloqueador da GP IIb/IIIa que tem sido mais extensivamente estudado. $\mathrm{O}$ abciximab é derivado do anticorpo monoclonal $m 7 \mathrm{E} 3$ de origem murina $(\mathrm{m})$. O fragmento Fc (fragmento cristalizável) é removido do anticorpo por digestão enzimática com papaína, resultando no m7E3 $\mathrm{Fab}^{53,59,60}$. Com a remoção do fragmento Fc, é minimizada a captura, pelo sistema reticuloendotelial, das plaquetas aderidas ao anticorpo, reduzindo o potencial para o desenvolvimento de trombocitopenia ${ }^{61}$. Com a finalidade de reduzir o potencial imunogênico, regiões constantes do anticorpo monoclonal murino são substituídas por seqüências de aminoácidos correspondentes à imunoglobulina humana, resultando no componente quimérico (c) humano-murino c7E3 Fab (fig. 1).

O abciximab atua ligando-se aos receptores plaquetários GP IIb/IIIa, prevenindo a ligação de outras moléculas ${ }^{23,38}$. Esse anticorpo monoclonal também bloqueia o receptor $\alpha v \beta_{3}$, que interage com a vitronectina ${ }^{34}$. Embora este receptor não tenha sido considerado quando o anticorpo foi inicialmente desenvolvido, esta reatividade cruzada tem provado ser benéfica na prevenção da agregação plaquetária ${ }^{62,63}$. Obloqueio o receptor $\alpha v \beta_{3}$ pode também inibir a migração e proliferação de células musculares lisas vasculares. Este efeito está potencialmente relacionado com a manutenção dos efeitos benéficos do abciximab a longo prazo. $\mathrm{O}$ abciximab, também inibe o inibidor 1 do ativador do plasminogênio (PAI-1) e o fator XIII. Em adição, liga-se ao receptor MAC-1 em monócitos ativados e estimula a retração do coágulo sangüíneo ${ }^{64,65}$. Devido ao fato que o coeficiente de ligação deste anticorpo com o receptor GP IIb/IIIa é muito maior do que aquele para o fibrinogênio, o abciximab tem o potencial

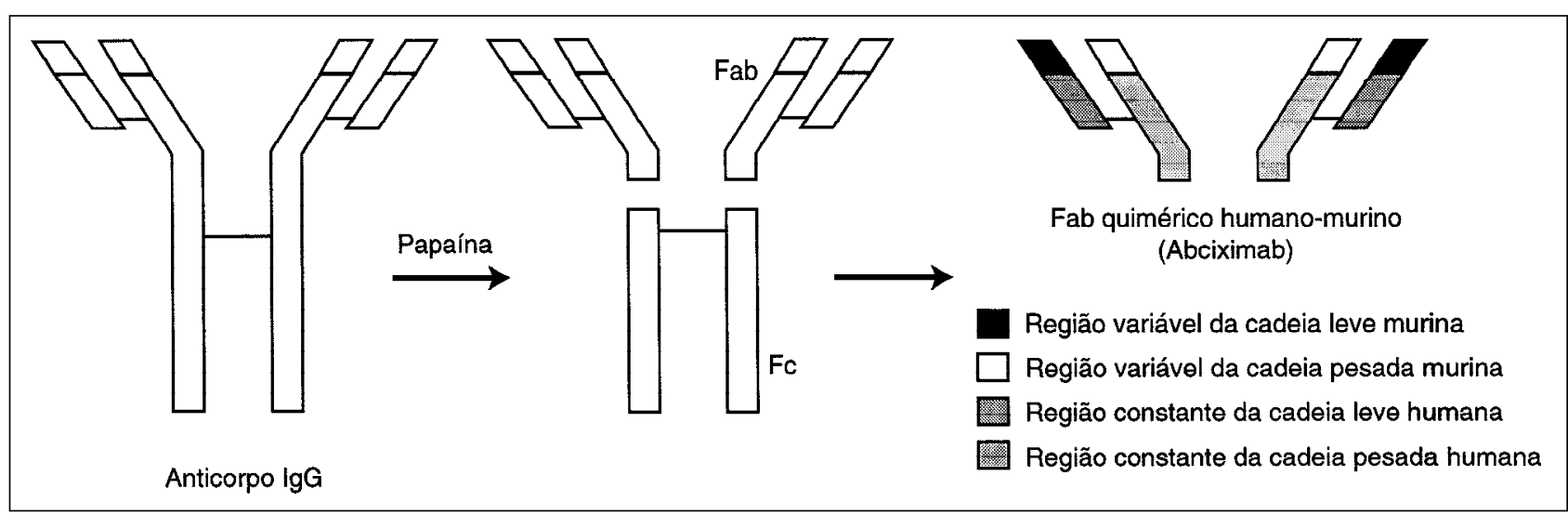

Fig. 1 - Anticorpo monoclonal c7E3 Fab ou abciximab. A digestão enzimática com papaína do anticorpo m7E3 produz os fragmentos Fab e Fc. As regiões constantes das cadeias leve e pesada do fragmento Fab murino são substituídas por seqüências de aminoácidos correspondentes à imunoglobulina humana, enquanto as regiões variáveis (responsáveis pela imunogenicidade) são mantidas, resultando no componente quimérico. 
para dissolver coágulos por desalojar fibrina e quebrar as pontes que ligam as plaquetas umas às outras ${ }^{66}$. O bloqueador GP IIb/IIIa pode ter esse efeito lítico em coágulos recentes. Os efeitos sobre um coágulo bem organizado, com mais de 48 h de evolução, é mínimo ${ }^{67,68}$.

Seguindo a administração intravenosa de abciximab, o efeito inibitório na função plaquetária é evidente dentro de $10 \mathrm{~min}{ }^{69}$. Após uma dose única, os níveis plasmáticos diminuem muito rapidamente, com uma meia vida inicial de menos de $10 \mathrm{~min}$, e uma $2^{\mathrm{a}}$ meia vida de, aproximadamente, $30 \mathrm{~min}^{6}$. Este rápido desaparecimento do plasma é, provavelmente, relacionado à rápida ligação com o receptor GP IIb/IIIa. Abciximab provoca inibição da função plaquetária dose-dependente. Após um bolus intravenoso de 0,25mg/ kg de abciximab, a agregação plaquetária induzida por ADP é completamente inibida. Esse efeito é sustentado por $12 \mathrm{~h}$ com a infusão intravenosa de $10 \mu \mathrm{g} / \mathrm{min}^{69}$. O abciximab permanece em circulação por vários dias num estado ligado a plaquetas, porém a função plaquetária global recupera-se ao longo de 48h. Nas doses recomendadas, o abciximab tem mínimo efeito no tempo de sangramento ${ }^{70,71}$.

\section{Estudos clínicos randomizados com abciximab}

Até o momento, três estudos clínicos randomizados, placebo controlados e duplo-cegos, foram completados, avaliando o uso do abciximab em mais de 6.000 pacientes submetidos a intervenções coronárias. Esses estudos foram concebidos para testar a hipótese que o bloqueio do receptor GP IIb/IIIa reduziria as complicações isquêmicas da angioplastia. Durante uma angioplastia, a lesão causada pela intervenção coronária rompe o endotélio sobre uma placa arterosclerótica e iatrogenicamente produz um trombo plaquetário ${ }^{72,73}$. Previamente, a disponibilidade dos inibidores da GP IIb/IIIa e do uso generalizado de stents, oclusão coronária aguda, uma das complicações mais devastadoras da angioplastia, ocorria em 4 a $8 \%$ dos procedi$\operatorname{mentos}^{74,75}$.

\section{EPIC}

O primeiro destes estudos foi o EPIC (Evaluation of c7E3 Fab in the Prevention of Ischemic Complications), um estudo que envolveu 2.099 pacientes de alto risco a serem submetidos à intervenções coronárias ${ }^{7}$. Esse estudo recrutou pacientes com síndromes coronárias isquêmicas agudas, ou seja, infarto do miocárdio (IM) recente ou angina pós-infarto, ou angina de repouso. Adicionalmente, foram incluídos pacientes com lesões coronárias complexas, tipo $\mathrm{B}_{2}$ ou C, como definido pela American Heart Associatione American College of Cardiology. Os pacientes foram randomizados para um dos seguintes tratamentos: 1) bolus e infusão por $12 \mathrm{~h}$ de placebo; 2) um bolus de abciximab $(0,25 \mathrm{mg} / \mathrm{kg})$, seguido por uma infusão de placebo por $12 \mathrm{~h}$; 3 ) bolus $(0,25 \mathrm{mg} / \mathrm{kg})$ e infusão $(10 \mu \mathrm{g} / \mathrm{min})$ de abciximab por 12h. Todos os pacientes receberam aspirina. Heparina foi administrada durante o procedimento para atingir tempos de coagulação ativados entre 300 e 350 s, e foi continuada como infusão por $12 \mathrm{~h}$. Os introdutores arteriais foram removidos $6 \mathrm{~h}$ após a heparina ser descontinuada, ou seja, $18 \mathrm{~h}$ após a intervenção. O desfecho primário (associação de morte, IM, ou nova revascularização dentro de 30 dias) ocorreu em $12,8 \%$ no grupo placebo, $11,4 \%$ nos pacientes recebendo apenas bolus de abciximab $(\mathrm{p}=0,43)$ e $8,3 \%$ nos pacientes que receberam bolus e infusão $(\mathrm{p}=0,008)$. Houve redução significativa não apenas no desfecho primário composto, mas também na incidência de IM e angioplastia urgente nos pacientes que receberam abciximab em bolus mais infusão (fig. 2).

Os resultados do estudo EPIC demonstraram que o bloqueio da GP IIb/IIIa com bolus e infusão de abciximab resultou numa redução absoluta de $4.5 \%$ e relativa de $35 \%$ no desfecho composto em 30 dias. Esta diferença foi mantida, ou mesmo aumentada, ao longo do tempo. Aos seis meses de seguimento, havia uma redução absoluta de $8 \%$ nessas complicações ${ }^{8}$. Contudo, os pacientes que receberam o inibidor plaquetário tiveram maior incidência de sangramento relacionado ao sítio de acesso arterial e receberam mais transfusões. Análise retrospectiva dos dados indicou que as complicações relacionadas a sangramento foram muito maiores em pacientes de baixo peso do que em pacientes mais pesados. Dessa forma, o sangramento excessivo foi atribuído à administração de heparina não ajustada para peso ${ }^{76}$.

$\mathrm{O}$ estudo EPIC validou a hipótese de que o bloqueio do receptor GP IIb/IIIa com abciximab, significativamente, reduz eventos isquêmicos agudos associados a angioplastia coronária de alto risco, a despeito de um aumento nas complicações hemorrágicas. Foi demonstrado que a fim de que se obtenha eficácia, abciximab deveria ser administrado em bolus, seguido de infusão contínua por um período de $12 \mathrm{~h}$.

O seguimento de longo prazo do estudo EPIC demonstrou que abciximab, administrado no momento da angioplastia coronária, teve eficácia prolongada ${ }^{77}$. Após 3 anos, o desfecho composto ocorreu em $41 \%$ dos que receberam

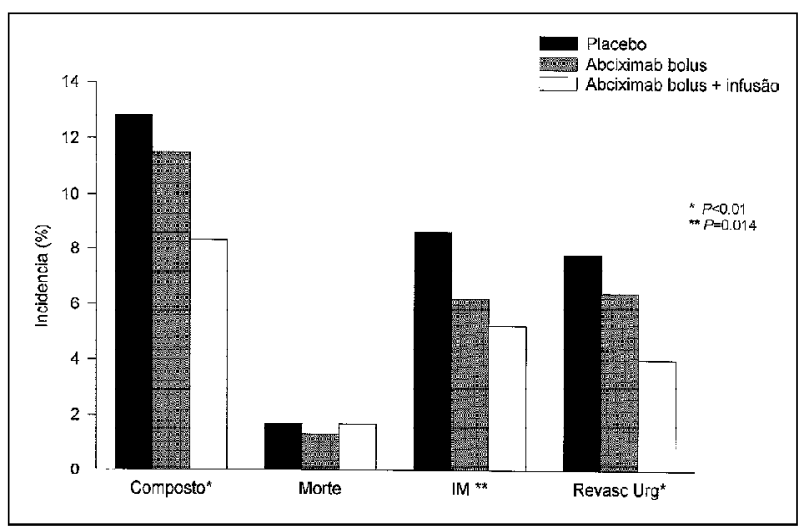

Fig. 2 - Estudo EPIC - eventos aos 30 dias nos grupos placebo e abciximab (bolus e bolus + infusão); composto - denota o desfecho primário composto de morte, infarto do miocárdio, e revascularização urgente. Valores de P referentes à comparação entre placebo e bolus + infusão. IM - infarto do miocárdio; Revasc Urg - revascularização urgente. 
tratamento ativo e em $47 \%$ dos que receberam placebo $(\mathrm{p}=0,009)$. Este foi o primeiro estudo clínico a demonstrar que uma intervenção farmacológica que objetiva reduzir complicações peri-angioplastia, pode produzir melhora sustentada nos resultados do procedimento. Esses dados levantaram a hipótese de que o bloqueio dos receptor GP IIb/IIIa determinaria estabilização da placa aterosclerótica. Este potencial efeito do abciximab, recentemente denominado "passivação arterial", implica na transformação da superfície vascular de uma situação que predispõem a deposição de plaquetas e trombose para uma situação estável.

\section{EPILOG}

O estudo EPILOG foi desenvolvido para responder as duas principais questões remanescentes do estudo EPIC: 1) seriam os efeitos salutares do abciximab evidentes em pacientes de baixo risco? 2) poderia o risco de sangramento ser reduzido com a utilização de heparina ajustada pelo peso? O desenho original deste estudo previa que 4.800 pacientes submetidos a angioplastia coronária fossem randomizados para receber placebo ou abciximab, associados a heparina em doses convencionais ou em doses baixas, ajustadas pelo peso ${ }^{78}$. Os grupos de tratamento incluíram: 1) placebo e bolus de heparina de $100 \mathrm{U} / \mathrm{kg}$, com a intenção de obter um tempo de coagulação ativada entre 300350s; 2) bolus e infusão de abciximab (similar às doses do estudo EPIC) associados a 100U/kg de heparina; 3) bolus e infusão de abciximab, como no EPIC, associados a 70U/kg de heparina, objetivando tempos de coagulação ativada entre 200-300s. Todos os pacientes foram tratados com aspirina. Heparina foi descontinuada, imediatamente após, a angioplastia e os introdutores arteriais foram removidos tão logo o tempo de coagulação ativada fosse inferior a 175 s. $\mathrm{O}$ protocolo do estudo solicitava que os investigadores obtivessem acesso arterial através de punção única da parede anterior do vaso e que a inserção de introdutores venosos fosse evitada. Pacientes de alto e baixo risco foram randomizados. Os únicos pacientes excluídos foram aqueles com o infarto agudo do miocárdio ou com angina instável associada a alterações eletrocardiográficas. Esses pacientes foram excluídos porque foi considerado não ser ético não tratá-los com abciximab.

O estudo EPILOG foi encerrado precocemente devido a eficácia do tratamento, após 2.792 pacientes terem sido randomizados. A análise dos resultados demonstrava uma redução de 56\% no desfecho composto aos 30 dias (morte, IM ou revascularização urgente) nos pacientes que receberam abciximab com heparina em baixas doses ou em doses convencionais ( $\mathrm{p}<0,001)$ (fig. 3). Aos seis meses, a análise combinada dos pacientes que receberam abciximab demonstrava uma redução relativa de $13 \%$ no desfecho composto de morte, infarto ou revascularização do miocárdio ( $\mathrm{p}=0,023$ ), e uma redução relativa de $43 \%$ no desfecho composto de morte, IM ou revascularização urgente $(\mathrm{p}<0,001)$. No grupo tratado com abciximab e baixas doses de heparina, não houve aumento na incidência de sangramento. Em rela-

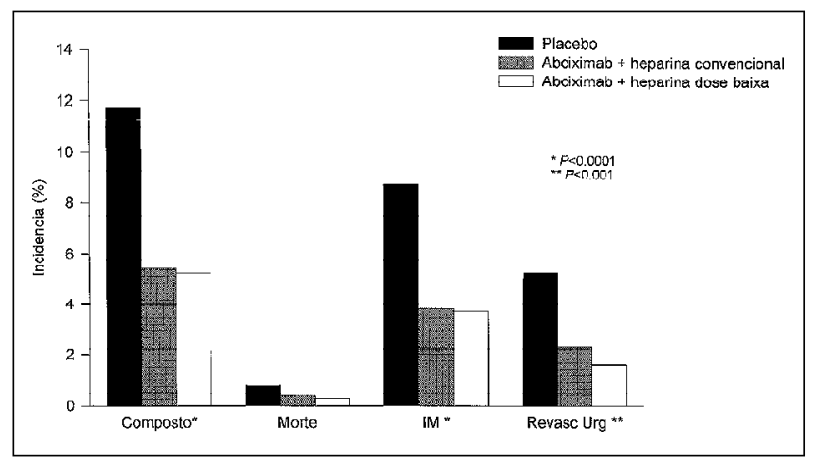

Fig. 3 - Estudo EPILOG - eventos aos 30 dias nos grupos placebo e abciximab (heparina em dose baixa ou convencional); composto - denota o desfecho primário composto de morte, infarto do miocárdio e revascularização urgente. Valores de P referentes a comparações entre placebo e abciximab + heparina em doses baixas. IMinfarto do miocárdio; Revasc Urg- revascularização urgente.

ção aos pacientes que receberam abciximab com doses convencionais de heparina, houve uma redução relativa de $35 \%$ nas complicações hemorrágicas.

A hipótese do bloqueio da GP IIb/IIIa foi confirmada no estudo EPILOG. O abciximab foi efetivo em pacientes de alto e baixo risco, submetidos a intervenção coronária. Houve significativas reduções na incidência de eventos compostos em um espectro de pacientes mais amplo, que incluía pacientes com uma semana de IM e pacientes com angina estável ou instável. De fato, houve uma maior eficácia do abciximab neste grupo de pacientes do que nos pacientes de alto risco no estudo EPIC. Em adição, o uso de heparina em doses ajustadas pelo peso, não seguido de infusão contínua e associado à remoção precoce do introdutor arterial, reduziu os riscos de sangramentos, aumentando a segurança do uso do abciximab.

\section{CAPTURE}

O terceiro estudo fase III com abciximab, ou CAPTURE (c7E3 Antiplatelet Therapy in Unstable Refractory Angi$n a$ ), foi limitado a pacientes com angina instável refratária ${ }^{79}$. Para serem randomizados, os pacientes tinham que ter tido pelo menos um episódio de dor torácica associado com modificações eletrocardiográficas compatíveis com isquemia miocárdica, dentro das últimas $48 \mathrm{~h}$, enquanto sob tratamento com heparina e nitroglicerina. Cineangiocoronariografia era executada dentro de $24 \mathrm{~h}$ da inclusão do paciente. Se a lesão relacionada à síndrome coronária era tratável por angioplastia, os pacientes eram randomizados para abciximab ou placebo. Abciximab foi administrado como um bolus de $0,25 \mathrm{mg} / \mathrm{kg}$ e infusão de $10 \mu \mathrm{g} / \mathrm{min}$ por $18-26 \mathrm{~h}$ antes da intervenção coronária. A infusão era mantida por apenas uma hora após a angioplastia.

O estudo CAPTURE, a exemplo do EPILOG, foi interrompido antes de ter sido completado (após 1.265 dos 1.400 pacientes terem sido randomizados), devido à significativa eficácia do abciximab. O desfecho primário em 30 dias, composto de morte, infarto ou revascularização do miocárdio urgente, foi significativamente reduzido no grupo abcixi- 
mab. A incidência destes eventos foi reduzida de 15,9\% para $11,3 \%$, uma redução relativa de $29 \%(\mathrm{p}=0,012)$. Em seis meses, contudo, houve uma aparente perda na eficácia do abciximab. A incidência de morte, intervenção percutânea ou revascularização cirúrgica coronária foram similares nos dois grupos. A conclusão obtida do estudo CAPTURE foi que o tratamento com abciximab reduziu eventos trombóticos em pacientes com angina instável refratária em $24 \mathrm{~h}$ e em 30 dias. Não houve efeito sustentado após esse período, assim como, não houve efeito sobre a reestenose.

\section{Abciximab na angina instável e no infarto agu- do do miocárdio}

O papel do abciximab em síndromes coronárias agudas e pacientes não submetidos a intervenções coronárias ainda encontra-se sob investigação. As informações disponíveis a respeito do uso de abciximab na angina instável são oriundas do estudo CAPTURE ${ }^{79}$. Um dos mais interessantes achados desse estudo foi que a diminuição dos eventos iniciou-se enquanto a droga estava sendo administrada, ou seja, antes da intervenção coronária. Isto é consistente com os resultados preliminares da inibição da GP IIb/IIIa com tirofibam em pacientes com angina instável nos estudos PRISM $^{80}$ e PRISM-PLUS ${ }^{81}$. Nesses estudos houve redução significante na incidência de eventos adversos em $48 \mathrm{~h}$ e em sete dias. Estas observações sugerem um potencial benefício da inibição da GP IIb/IIIa na angina instável.

A formulação murina do 7E3 (m7E3) foi, inicialmente, estudada em 60 pacientes com infarto agudo do miocárdio tratados com terapia convencional que incluía o uso do ativador do plasminogênio de tecidual recombinante, ácidoacetilsalicílico e heparina ${ }^{10}$. A comparação dos resultados dessa série de casos com um grupo controle sugeria a redução no número de eventos isquêmicos em pacientes tratados com o m7E3. Resultados iniciais de outros estudos também sugerem que a administração dos bloqueadores da GP IIb/IIIa no IM seja benéfica ${ }^{11,18}$. Atualmente, a utilização do abciximab em associação com doses reduzidas de trombolíticos no manejo do IM está sendo avaliado pelo estudos TIMI 14 e GUSTO 4.

\section{Estudos clínicos randomizados com outros inibidor da GP IIb/IIIa}

Vários inibidores competitivos do receptor GP IIb/IIIa têm sido testados em estudos clínicos randomizados, duplo-cegos e placebo controlados envolvendo um grande número de pacientes. O estudo IMPACT II ${ }^{82}$ avaliou o uso do eptifibatide em mais de 4.000 pacientes submetidos a angioplastia. Os estudos RESTORE ${ }^{83}$, PRISM $^{80}$ e PRISM PLUS ${ }^{81}$ randomizaram mais de 7.000 pacientes, para testar a eficácia do pseudo-peptídeo tirofibam na redução das complicações isquêmicas da angioplastia em pacientes instáveis e no manejo da angina instável e do infarto subendocárdico. $\mathrm{O}$ estudo PARAGON ${ }^{17}$ avaliou o lamifibam em mais de 2.000 pacientes com angina instável ou infarto subendocárdico.
O IMPACT II (Integrilin to Manage Platelet Aggregation to Prevent Coronary Thrombosis), incluiu 4.010 pacientes submetidos a angioplastia coronária urgente e eletiva ${ }^{82}$. Dois regimes de eptifibatide foram utilizados: um bolus $135 \mu \mathrm{g} / \mathrm{kg}$ e infusão de $0,5 \mu \mathrm{g} / \mathrm{kg} / \mathrm{min}$ ou de $0,75 \mu \mathrm{g} / \mathrm{kg} /$ min, administrado por $20 \mathrm{a} 24 \mathrm{~h}$. Essas infusões foram comparadas com bolus e de infusão de placebo. Todos os pacientes receberam ácido-acetilsalicílico e heparina ajustados pelo peso para obter um tempo de coagulação ativada acima de 300s. O eptifibatide foi efetivo na prevenção de eventos isquêmicos durante as primeiras $24 \mathrm{~h}$ após o procedimento. O desfecho composto de morte, IM, revascularização de urgência, ou implante de stents por oclusão aguda foi significativamente reduzido de $11,9 \%$ para $6,8 \%$ nos grupos que receberam eptifibatide, uma redução relativa de $29 \%$. Aos 30 dias houve uma redução relativa de $19 \%$ no desfecho composto no grupo que recebeu infusão de baixas doses $(11,4 \%$ vs $9,2 \%$; $p=0,065)$ e de $13 \%$ no grupo que recebeu doses maiores $(11,4 \%$ vs $9,9 \% ; p=0,22)$. A pequena redução na incidência de eventos adversos combinada aos 30 dias nos grupos tratados com eptifibatide foi preservado aos seis meses, mas nenhum benefício adicional foi observado. Isto foi consistente com o subestudo angiográfico que não demonstrou diferenças no diâmetro luminal coronário mínimo ou na incidência de reestenose.

O uso de tirofibam foi avaliado no estudo RESTORE (Randomized Efficacy Study of Tirofibam for Outcomes and Restenosis), em pacientes com síndromes coronárias agudas submetidos à angioplastia ${ }^{83}$. Nesse estudo, 2.139 pacientes foram randomizados dentro de $72 \mathrm{~h}$ de um IM ou de um episódio de angina instável. Tirofibam foi administrado como um bolus de $10 \mu \mathrm{g} / \mathrm{kg}$ seguido de uma infusão de $0,15 \mu \mathrm{g} / \mathrm{kg} / \mathrm{min}$ por $36 \mathrm{~h}$. Houve significante reduções na incidência de eventos em 48h (38\%) e em sete dias (27\%). Contudo, de modo similar ao IMPACT II, este efeito foi reduzido em 30 dias. O desfecho composto de morte, ou IM, ou revascularização urgente dentro de 30 dias foi reduzido de $12,2 \%$ para $10,3 \%$. Esta redução relativa de $16 \%$ nos pacientes que receberam a droga ativa não foi estatisticamente significativa.

Outros dois estudos de grande porte envolvendo o tirofibam foram completados até o momento. No estudo PRISM (Platelet Receptor Inhibition for Ischemic Syndrome Management) 3.231 pacientes com angina instável e IM subendocárdico foram randomizados para receber heparina ou tirofibam por 48 horas $^{80}$. Ao final destas 48 horas, o desfecho composto (morte, IM e angina refratária) foi reduzido com tirofibam (3,8 vs 5,9\%, p<0,05). Em 30 dias, o benefício inicial foi parcialmente perdido ( $12,8 \mathrm{vs} 13,9 \%)$, contudo a mortalidade no grupo tirofibam era menor $(2,3$ vs $3,6 \%$, $\mathrm{p}<0,02)$. Não houve aumento significativo na incidência de sangramentos maiores em pacientes tratados com tirofibam. No estudo PRISM-PLUS (Platelet Receptor Inhibition for Ischemic Syndrome Management in Patients Limited by very Unstable Signs and Symptoms) o uso do tirofibam foi avaliado no manejo clínico e intervencionista em 1.815 pacientes com angina instável e infarto subendocárdico ${ }^{81}$. Os 
pacientes foram originalmente randomizados para um dos três tratamentos possíveis: tirofibam isolado, heparina isolada ou heparina mais tirofibam. O grupo tirofibam isolado foi precocemente descontinuado devido a um excesso de eventos. Os pacientes recebiam uma infusão de $48 \mathrm{~h}$ da droga em estudo e, então, poderiam ser submetidos a angiografia coronária. Se a angiografia não era realizada, a droga em estudo era descontinuada. Se uma intervenção coronária era executada, tirofibam era infundido por $12 \mathrm{~h}$ adicionais. Aos sete dias, a incidência do desfecho composto foi reduzida significativamente no grupo que recebia heparina mais tirofibam (12,9 vs 17,9\% no grupo heparina isolada). Aos 30 dias, a evidência de benefício era menos marcada (18,5\% vs $22,3 \%$ ). Novamente, não houve aumento significativo na incidência de sangramentos com tirofibam.

OestudoPARAGON (Platelet IIb/IIIa Antagonist for the Reduction of Acute Coronary Syndrome events in a Global Organization Network) ${ }^{17}$ avaliou a administração de lamifibam em 2.282 pacientes com angina instável ou infarto subendocárdico. Aos 30 dias, não houve redução significativa na incidência do desfecho composto com a administração de lamifibam (10,3\% vs 11,7\%). Contudo, aos seis meses, houve uma redução altamente significativa, de aproximadamente $40 \%$ na incidência de morte ou IM no grupo que recebeu lamifibam em doses baixas quando comparado com placebo.

A falha da manutenção a médio prazo do efeito clínico benéfico inicial observado em alguns estudos com os inibidores competitivos do GP IIb/IIIa têm sido atribuídos à dosagem ou duração da administração da droga inadequados.

\section{Abciximab e stents}

Desde a publicação dos estudos multicêntricos STRESS ${ }^{84}$ e BENESTENT ${ }^{85}$, a utilização de stents tem aumentado progressivamente. A despeito dos resultados favoráveis iniciais, o uso de stents permaneceu limitado devido a uma incidência de oclusão subaguda de 3 a 5\% e da necessidade de anticoagulação intensiva. Isto foi mudado, dramaticamente, após Colombo e col ${ }^{86}$, baseados em observações por ultra-som intravascular, relatarem que a maioria dos stents não expandia completamente a não ser que altas pressões fossem utilizadas no seu posicionamento. A completa expansão do stent com altas pressões otimiza o fluxo sangüíneo coronário, diminuindo significativamente as chances de oclusão subaguda ${ }^{87}$. Isto permitiu que a anticoagulação pós-stent fosse dispensada, sendo mantidos

\section{Composto}

Morte / IM / TVR

IM

\section{Angioplastia Urgente}

TVR

\section{RCM Urgente}

RCM

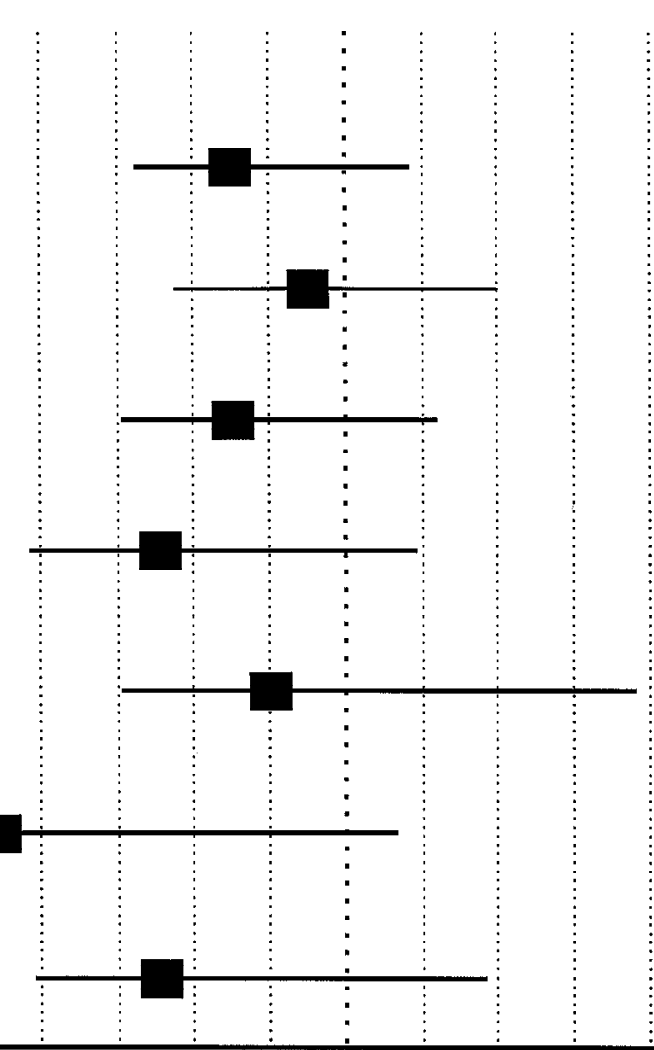

\section{5}

\section{Placebo}

20.8

8.1

c7E3

\section{$20.8 \quad 10.1$}

$17.3 \quad 6.5$

$\begin{array}{ll}7.7 & 2.0\end{array}$

$8.6 \quad 3.9$

$4.5 \quad 0.3$

Fig. 4 - Metanálise do subgrupo de pacientes que receberam stents nos estudos EPIC, EPILOG e CAPTURE. Risco relativo e intervalo de confiança (95\%) para os eventos em 30 dias nos grupos abciximab combinados (heparina em dose baixa + convencional) comparados ao grupo placebo. Os valores à direita representam a incidência de eventos; composto - denota o desfecho primário composto de morte, infarto do miocárdio, e revascularização urgente. IM- infarto do miocárdio; TVR- revascularização do vaso previamente intervido; RCM- revascularização cirúrgica do miocárdio. 
apenas os agentes antiplaquetários, normalmente ácidoacetilsalicílico e ticlopidina ${ }^{88,89,90}$. Estes refinamentos na técnica de implantação de stents reduziram significativamente a incidência de oclusão subaguda, sangramento arterial e complicações vasculares. Com isso, a implantação de stents aumentou rapidamente para mais de $50 \%$ em vários laboratórios de cardiologia intervencionista ${ }^{91,92}$. No estudo BENESTENT II ${ }^{93}$, angioplastia coronária convencional foi comparada com o uso de stents Palmaz-Schatz cobertos com heparina, implantados com altas pressões, associados ao ácido-acetilsalicílico e ticlopidina. A incidência de complicações agudas com stent foi extremamente baixa e não houve diferença na incidência de sangramento ou complicações vasculares entre os dois procedimentos.

O posicionamento de stents tem demonstrado ser um tratamento efetivo não apenas para oclusão coronária aguda pós-angioplastia, mas também como terapia primária. Apesar de que as razões para implante destents sejam bastante dependentes do médico executando o procedimento, stents são geralmente utilizados em pacientes que, após angioplastia com balão, apresentam lesões residuais que limitam o fluxo sangüíneo ou dissecções complexas ${ }^{94-97}$. Stents também são utilizados quando um resultado obtido com o balão é inadequado ( $>30 \%$ de estenose residual $)^{98} \mathrm{e}$ para lesões com morfologia desfavorável, nas quais a angioplastia com o balão torna-se improvável de produzir resultados adequados, como em pontes safenas ${ }^{99-101} \mathrm{ou}$ oclusões crônicas ${ }^{102-105}$. Stents também são, freqüentemente, utilizados para tratar lesões reestenóticas ${ }^{106}$ e em casos onde a morbidade ou mortalidade cirúrgica é aumentada ${ }^{107-108}$. Finalmente, stents têm sido utilizados mais frequientemente para dilatação de lesões primárias, particularmente, de estenoses proximais na artéria descendente anterior esquerda ${ }^{110}$.

Uma vez que stents e abciximab foram desenvolvidos recentemente, não há resultados disponíveis de estudos comparando estas duas modalidades terapêuticas. Apesar de que abciximab seja um agente antiplaquetário muito efetivo é difícil determinar se ele complementaria o posicionamento de stents, considerando-se que a incidência de oclusão aguda de stents posicionados com altas pressões é inferior a $2 \%{ }^{86,93}$. Nos pacientes que foram submetidos à implantação eletiva de stent nos estudos EPILOG ${ }^{78}$ e CAPTURE $^{79}$, a incidência do desfecho composto aos 30 dias (morte, IM, ou revascularização de urgência) foi 11,7\% no grupo placebo e 6,6\% nos que receberam abciximab ${ }^{111}$. Esta redução relativa de $44 \%$ nos efeitos adversos não atinge significância estatística, devido ao pequeno número de pacientes. Nos 326 pacientes que receberam stents não planejados no estudo EPILOG ${ }^{112}$, o desfecho composto aos 30 dias foi reduzido em mais de $60 \%$ nos pacientes que receberam a droga ativa. A necessidade de cirurgia coronária de urgência foi virtualmente abolida (5,7\% no grupo placebo comparado com $1,2 \%$ no grupo abciximab mais heparina em baixas doses e $0 \%$ no grupo abciximab mais heparina em doses convencionais). A melhora no desfecho composto em seis meses, nos pacientes randomizados para abciximab que receberam stents foi similar ou melhor do que a obtida nos demais pacientes. O resultado da meta-análise do subgrupo de pacientes que recebeu stents nos estudos EPIC, EPILOG e CAPTUREé apresentado na figura 4.

A maior razão para não se utilizar abciximab, na maioria dos paciente submetidos a revascularização percutânea, é o custo da droga, que atualmente é de, aproximadamente, $\mathrm{R}$ \$ 1.600,00 por caso. Devido às restrições econômicas, muitos intervencionistas prefeririam usar stents, que hoje custam, aproximadamente, R\$1.500,00, a administrar abciximab. Este raciocínio geral tem como premissa a percepção de que os resultados obtidos com os stents são tão bons, que abciximab não seria necessário na maioria dos pacientes. Entretanto, os resultados do recentemente publicado EPISTENT demonstram que a administração de abciximab reduz significantemente a incidência de eventos isquêmicos em pacientes submetidos a implante de stents. Neste estudo, 2.399 pacientes de alto e baixo risco foram randomizados para receber apenas implante de stent, com abciximab, ou angioplastia com balão com abciximab. Quando comparados ao grupo que recebeu apenas stent, houve uma redução significativa no desfecho composto aos 30 dias (morte, infarto do miocárdio, ou revascularização de urgência) de $51 \%$ no grupo stent com abciximab e de $36 \%$ no grupo balão com abxicimab ${ }^{113}$.

\section{Conclusão}

Os estudos, avaliando o anticorpo monoclonal contra a GP IIb/IIIa, demonstraram que a terapia com abciximab foi efetiva na redução de eventos adversos em, praticamente, todos os tipos de pacientes submetidos a angioplastia coronária. Isto inclui pacientes com angina refratária ${ }^{79}, \mathrm{com}$ síndromes coronárias isquêmicas agudas ou lesões de morfologia complexa ${ }^{7}$ e submetidos a angioplastia coronária eletiva ${ }^{78}$. A redução na incidência de eventos adversos em 30 dias foi superior a $50 \%$ com o tratamento ativo. Esses resultados têm se mostrado sustentados ao longo do tempo ${ }^{77}$.

Com base nos estudos clínicos publicados, o uso do anticorpo monoclonal contra glicoproteína plaquetária IIb/ IIIa deveria ser considerado em qualquer paciente submetido a uma intervenção coronária percutânea. Limitações impostas pelo elevado custo poderiam determinar que a associação stents-abciximab fosse reservada para casos complexos ou de risco elevado, restringindo o uso de abciximab a pacientes que possuissem um maior benefício absoluto. Abciximab provavelmente deveria ser usado em associação com stents em pacientes com angina instável refratária e IM recente ou com trombo coronário angiograficamente visível. Outra razão potencial para o uso do abciximab com stents seria a presença de uma dissecção coronária extensiva ou incompletamente coberta pelo stent, o posicionamento de stents em vasos difusamente ateroscleróticos, assim como o implante de múltiplos stents. Embora no estudo EPILOG, o abciximab não tenha reduzido a reestenose clínica, após angioplastia com balão (revascularização da coronária previamente intervida), é possível que os inibidores do 
receptor GP IIb/IIIa reduzam reestenose pós-stent, uma vez que a fisiopatogenia da reestenose pós-stent difere, consideravelmente, sendo, fundamentalmente, o resultado de extensiva hiperplasia intimal ${ }^{114}$

A administração dos bloqueadores da GP IIb/IIIa no manejo da angina instável e do IM é bastante promissora, com base nos resultados dos estudos PARAGON ${ }^{17} \mathrm{e}$ CAPTURE $^{79}$. Contudo, ainda persistem dúvidas consideráveis a respeito da dosagem e duração do tratamento para os diversos agentes. Uma melhor avaliação das indicações dos bloqueadores da GP IIb/IIIa nas síndromes coronárias ins- táveis em pacientes não submetidos a angioplastia coronária deverá ser realizada com a publicação dos resultados finais de vários estudos, recentemente concluídos, ou em andamento. O uso dos bloqueadores da GP IIb/IIIa associado com terapia trombolítica no IM ainda é incerto. Estudos de grande porte atualmente em andamento fornecerão informações definitivas em relação a eficácia e risco de sangramento vinculado ao uso desta associação. Finalmente, o desenvolvimento de bloqueadores da GP IIb/IIIa para uso oral poderá se constituir num grande avanço no manejo das diversas síndromes clínicas da cardiopatia isquêmica.

\section{Referências}

1. Lefkovitz J, Plow EF, Topol EJ - Platelet glycoprotein IIb/IIIa receptors in cardiovascular medicine. N Engl J Med 1995; 332: 1553-9.

2. Lefkovitz J, Topol EJ - Platelet glycoprotein IIb/IIIa receptor antagonists in coronary artery disease. Eur Heart J 1996; 17: 9-18.

3. Tcheng JE - Platelet Integrin Glycoprotein IIb/IIIa Inhibitors: Opportunities and Challenges. J Invas Cardiol 1996; 8(suppl B): 8B-14B.

4. Coller BS - Platelets and thrombolytic therapy. N Engl J Med 1990; 332: 33-42.

5. Ellis SG, Tcheng JE, Navetta FI et al - Safety and antiplatelet effect of murine monoclonal antibody 7EC Fab directed against platelet glycoprotein IIb/IIIa in patients undergoing elective coronary angioplasty. Coron Artery Dis 1993; 4: 167-75.

6. Tcheng JE, Ellis SG, George BS et al - Pharmacodynamics of chimeric glycoprotein IIb/IIIa integrin antiplatelet antibody Fab 7E3 in high-risk coronary angioplasty. Circulation 1994; 90: 1757-64.

7. The EPIC Investigations - Use of a monoclonal antibody directed against the platelet glycoprotein IIb/IIIa receptor in high-risk coronary angioplasty. N Engl J Med 1994; 330: 956-61.

8. Topol EJ, Califf RM, Weisman HF et al-Randomized trial of coronary intervention with antibody against platelet IIb/IIIa integrin for reduction of clinical restenosis:results at six months. Lancet 1994; 343: 881-6.

9. Simoons ML, De Boer MJ, Van Den Brand MJ et al-Randomized trial of a GP IIb/ IIIa platelet receptor blocker in refractory unstable angina. Circulation 1994; 89: 596-603.

10. Kleiman NS, Ohman EM, Califf RM et al - Profound Inhibition of Platelet Aggregation with Monoclonal Antibody 7E3 Fab after Thrombolytic Therapy. J Am Coll Cardiol 1993; 22: 381-9.

11. Cigarroa JE, Ferrell MA, Collea DJ, Leinbach RC - Enhanced endogenous coronary thrombolysis during acute myocardial infarction following selective platelet receptor blockade with ReoPro. Circulation 1996; 94: I-553.

12. Ohman EM, Harrington RA, Lincoff AM et al - Early clinical experience with integrelin, an inhibitor of the platelet glycoprotein IIb/IIIa integrin receptor. Eur Heart J 1995; 16(suppl L): 50-5.

13. Tcheng JE, Harrington RA - Multicenter, randomized, double-blind, placebocontrolled trial of the platelet integrin glycoprotein IIb/IIIa blocker integrelin in elective coronary intervention. Circulation 1995; 91: 2151-7.

14. Schulman SP, Goldschmidt-Clermont PJ, Topol EJ et al - Effects of integrelin, a platelet glycoprotein IIb/IIIa receptor antagonist, in unstable angina. Circulation 1996; 94: 2083-9.

15. Théroux P, Kouz S, Royl et al - Platelet membrane receptor glycoprotein IIb/IIIa antagonism in stable angina. The Canadian Lamifiban Study Circulation 1996; 94: 899-905.

16. Cannon CP, Novotny WF, McCabe $\mathrm{CH}$ et al - Evaluation of the oral glycoprotein IIb/IIIa antagonist R048-3657 in patients' post acute coronary syndromes:Primary results of the TIMI 2 Trial. Circulation 1996; 94: I-552.

17. PARAGON Investigators - A randomized trail of potent platelet IIb/IIIa antagonism, heparin, or both in patients with unstable angina: The PARAGON Study. Circulation 1996; 94: I-553.

18. Moliterno DJ, Harrington RA, Krucoff MW et al - More complete and stable reperfusion with platelet IIb/IIIa antagonism plus thrombolysis for AMI: The PARADIGM Trial. Circulation 1996; 94: I-553.

19. Harrington RA, Moliterno DJ, Van de Werf F et al - Delaying and preventing ischemia events in patients with acute coronary syndromes using the platelet glycoprotein IIb/IIIa inhibitor lamifiban. J Am Coll Cardiol 1997; 29: 409A.

20. Fernandez-Ortiz A, Badimon JJ, Falk E et al - Characterization of the relative thrombogenicity of atherosclerotic plaque components: implications for consequences of plaque rupture. J Am Coll Cardiol 1994; 23: 1562-9.
21. Coller BS - Platelets in Cardiovascular Thrombosis and Thrombolysis. In Fozzard HA, Haber E, Jennings RB et al - The Heart and Cardiovascular System: Scientific Foundations, eds. New York: Raven Press 1991; 219-73.

22. Leung L, Nachman R - Molecular mechanisms of platelet aggregation. Ann Rev Med 1986; 37: 179-86.

23. Plow EF, McEver RP, Coller BS et al - Related binding mechanisms for fibrinogen, fibronectin, von Willebrand factor and thrombospondin on thrombin-stimulated human platelets. Blood 1985; 66: 724-7.

24. Plow EF, D'Souza SE, Ginsberg MH - Ligand binding to GP IIb/IIIa:a status report. Sem Throm Hemo 1992; 18: 324-32.

25. Coller BS - Blood elements at surfaces:platelets. Annals of the New York Academy of Sci 1987; 516: 362-79.

26. Roberts HR, Lozier JN - New perspectives on the coagulation cascade. Hosp Pract 1992; 27: 97-105, 109-12.

27. Fuster V, Badimon L, Badimon JJ, Chesebro JH - The pathogenesis of coronary artery disease and the acute coronary syndromes. N Engl J Med 1992; 326: $242-50$.

28. Fuster V, Badimon L, Badimon JJ, Chesebro JH - The pathogenesis of coronary artery disease and the acute coronary syndromes(2). N Engl J Med 1992; 326: 310-8.

29. Phillips DR, Charo IF, Parise LV, Fitzgerald LA - The platelet membrane glycoprotein IIb/IIIa complex. Blood 1988; 71: 831-43.

30. Pytela R, Pierschbacher MD, Ginsberg MH, Plow EF, Rouslahti E - Platelet membrane glycoprotein IIb/IIIa: member of a family of Arg-Gly-Asp-specific adhesion receptors. Science 1986; 231: 1559-62.

31. Hynes RO - Integrins: Versatility, Modulation and Signalling in Cell Adhesion. Cell 1992; 69: 11-25.

32. Fitzgerald LA, Steiner B, Rall SC Jr, Lo SS, Phillips DR - Protein sequence of endothelial glycoprotein IIIa derived from a cDNA clone. J Biol Chem 1987; 262: 3936-9.

33. Poncz M, Eisman R, Heidenreich R et al - Structure of the platelet membrane glycoprotein IIb. J Biol Chem 1987; 262: 8476-82.

34. Felding-Habermann B, Cheresh DA - Vitronectin and its receptors. Curr Opin Cell Biol 1993; 5: 864-8.

35. Coller BS, Cheresh DA, Asch E, Seligsohn U - Platelet Vitronectin Receptor Expression Differentiaties Iraqi-Jewish from Arab Patients with Glanzmann Thrombasthenia in Israel. Blood 1991; 77: 75-83.

36. Weisel JW. Nagaswami C, Vilaire G, Bennett JS - Examination of the platelet membrane glycoprotein IIb/IIIa complex and its interaction with fibrinogen and other ligands by electron microscopy. J Biol Chem 1992; 23: 16637-43.

37. Ruoslahti E, Pierschbacher MD - Arg-Gly-Asp:a versatile cell recognition signal. Cell 1986; 44: 517-8.

38. Cheresh DA, Berliner SA, Vincente V, Roggeri ZM - Recognition of distinct adhesive sites on fibrinogen by related integrins on platelets and endothelial cells. Cell 1989; 58: 945-53.

39. D'Souza SE, Ginsberg MH, Plow EF - Arginyl-glycyl-aspartic acid(RGD) :a cell adhesion motif. Trends Bio Sci 1991; 16: 246-50.

40. Kloezewiak M, Timmons S, Hawiger J - Recognition site for platelet receptor is present on the 15-residue carboxy-terminal fragment of the gamma chain of human fibrinogen and is not involved in the fibrin polymerization reaction. Throm Res 1983; 29: 249-55.

41. Fox JE - The platelet cytoskeleton. Throm Hemo 1993; 70: 884-93.

42. Schwartz L, Bourassa MG, Lesperance J et al - Aspirin and dipyridamole in the prevention of restenosis after percutaneous transluminal coronary angioplasty. N Engl J Med 1988; 318: 1714-9. 
43. Barnathan ES, Schwartz JS, Taylor L et al - Aspirin and dipyridamole in the prevention of acute coronary thrombosis complicating coronary angioplasty. Circulation 1987; 76: 125-34.

44. Heras M, Chesebro JH, Penny WJ et al - Importance of adequate heparin dosage in arterial angioplasty in a porcine model. Circulation 1988; 78: 654-60.

45. Lukas MA, Deutsch E, Laskey WK-Beneficial effect of heparin therapy on PTCA outcome in unstable angina. J Am Coll Cardiol 1988; 11(suppl): 132A.

46. Pastrono C - Aspirin and human platelets:from clinical trials to acetylation of cyclooxygenase and back. Trends Pharm Sci 1989; 10: 453-8.

47. Willard JE, Lange RA, Hillis LD - The use of aspirin in ischemia heart disease. $\mathrm{N}$ Engl J Med 1992; 327: 175-81.

48. Pastrono C - Aspirin as an antiplatelet drug. N Engl J Med 1994; 330: 1287-94.

49. Brezinski DA, Nesto RW, Serhan CN - Angioplasty triggers intracoronary leukotrienes and lipokin A4. Impact of aspirin therapy. Circulation 1992; 86: 56-63.

50. Fitzgerald GA, Oates JA, Hawiger J et al - Endogenous biosynthesis of prostacyclin and thromboxane and platelet function during chronic administration of aspirin in man. J Clin Invest 1983; 71: 676-88.

51. Buchanan MR, Brister JJ - Individual variation in the effects of ASA on platelet function:implications for the use of ASA clinically. Can J Cardiol 1995; 11: 221-7.

52. Coller BS, Peerschke EI, Scudder LE, Sullivan CA - A murine monoclonal antibody that completely blocks the binding of fibrinogen to platelets produces a thrombasthenic-like state in normal platelets and binds to glycoproteins IIb and/or IIIa. J Clin Inv 1983; 72: 325-38.

53. Coller BS - A new murine monoclonal antibody reports an activation-dependent change in the conformation and/or microenvironment of the platelet glycoprotein Ilb/IIIa complex. J Clin Inv 1985; 76: 101-8.

54. Harrington RA, Kleiman NS, Kottke-Marchant K et al - Immediate and reversible platelet inhibition after intravenous administration of a peptide glycoprotein IIb/ IIIa inhibitor during percutaneous coronary intervention. Am J Cardiol 1995; 76: 1222-7.

55. Carteaux JP, Steiner B, Roux S - Ro 44-9883, a new non-peptidic GPIIb-GPIIIa antagonist prevents platelet loss in a guinea pig model of extracorporeal circulation. Thromb Haemost 1993; 70: 817-21.

56. Reveiakes DJ, Kleiman NS, Ambrose J - Randomized, double-blind, placebocontrolled dose-ranging study of tirofiban(MK-383) platelet IIb/IIIa blockade in high-risk patients undergoing coronary angioplasty. J Am Coll Cardiol 1996; 17: 536-42.

57. Nicholson NS, Panzer-Knodle SG, Salyers AK et al - SC-54684A:an orally active inhibitor of platelet aggregation. Circulation 1995; 91: 403-10.

58. Szalony JA, Haas NF, Salyers AK et al - Extended inhibition of platelet aggregation with the orally active platelet inhibitor SC-54684A. Circulation 1995; 91: 411-6.

59. Calvette JJ - Clues for understanding the structure and function of a prototypic human integrin:the platelet glycoprotein IIb/IIIa complex. Thromb Haemost 1994; 72: 1-15.

60. Coller BS, Folts JD, Scudder LE, Smith SR - Antithrombotic effect of a monoclonal antibody to the platelet glycoprotein IIb/IIIa receptor in an experimental animal model. Blood 1986; 68: 783-6.

61. Coller BS, Scudder LE - Inhibition of dog platelet function by in vivo infusion of $\mathrm{F}(\mathrm{ab}$ ')2 fragments of a monoclonal antibody to the platelet glycoprotein IIb/IIIa receptor. Blood 1985; 66: 1456-9.

62. Plow EF, Ginsberg MH - Cellular adhesion:GP IIb/IIIa as a prototype adhesion receptor. Prog Hemo Throm 1989; 9: 117-56.

63. Charo IF, Bekeart LS, Phillips DR - Platelet glycoprotein IIb/IIIa-like proteins mediate endothelial cell attachment to adhesive proteins and the extracellular matrix. J Biol Chem 1987; 262: 9935-8.

64. Hanson SR, Pareti FI, Ruggeri ZM et al - Effects of monoclonal antibodies against the platelet glycoprotein IIb/IIIa complex on thrombosis and hemostasis in the baboon. J Clin Invest 1988; 81: 149-58.

65. Ellis SG, Bates ER, Schaible T et al - Prospects for the use of antagonists to the platelet glycoprotein IIb/IIIa receptor to prevent post-angioplasty restenosis and thrombosis. J Am Coll Cardiol 17: 89B-95B.

66. Cohen I, Burk DL, White JG - The effect of peptides and monoclonal antibodies that bind to platelet glycoprotein IIb/IIIa complex on the development of clot tension. Blood 1989; 73: 1880-7.

67. Reverter JC, Beguin S, Kessels H et al - Inhibition of platelet-mediated, tissue factor-induced thrombin generation by the mouse/human chimeric 7E3 antibody. Potential implications for the effect of C7E3 Fab treatment on acute thrombosis. J Clin Inv 1996; 98: 863-74.

68. Gold HK, Coller BS, Yasuda T et al - Rapid and sustained coronary artery recanalization with combined bolus injection of recombinant tissue-type plasminogen activator and monoclonal antiplatelet GP IIb/IIIa antibody in a canine preparation. Circulation 1988; 77: 670-7.

69. Faulds D, Sorkin EM - Abciximab(c7E3 Fab):a review of its pharmacology and therapeutic potential in ischemic heart disease. Drugs 1994; 48: 583-98.
70. Coller BS, Folts JD, Smith SR, Scudder LE, Jordan R - Abolition of In Vivo Platelet Thrombus Formation in Primates with Monoclonal Antibodies to the Platelet GP IIb/IIIa Receptor: Correlation with Bleeding Time, Platelet Aggregation and Blockade of GP IIb/IIIa Receptors. Circulation 1989; 80: 1766-74.

71. Coller BS, Scudder LE, Beer J et al - Monoclonal Antibodies to Platelet GP IIb/ IIIa as Antithrombotic Agents. Ann N Y Acad Sci. 1991; 614: 193-213.

72. Waller BF - "Crackers, breakers, stretchers, drillers, scrapers, shavers, burners, welders and melters" - the future treatment of atherosclerotic coronary artery disease? J Am Coll Cardiol 1989; 13: 969-87.

73. Waller BF, Orr CM, Pinkerton CA et al - Coronary balloon angioplasty dissections:the good, the bad and the ugly. J Am Coll Cardiol 1992; 20: 701-6.

74. Holmes DR Jr, Holubkov R, Vlietstra RE et al - Comparison of complications during percutaneous transluminal coronary angioplasty from 1977 to 1981 and from 1985 to 1986. J Am Coll Cardiol 1988; 12: 1149-55.

75. Lincoff AM, Topol EJ - Abrupt Vessel Closure. In: Topol EJ - Textbook of Interventional Cardiology, ed. Philadelphia: WB Saunders, 1994: 207-30.

76. Aguirre FV, Topol EJ, Ferguson JJ et al - Bleeding complications with the chimeric antibody to platelet glycoprotein IIb/IIIa integrin in patients undergoing percutaneous coronary intervention. EPIC Investigators. Circulation 1995; 91: 2882-90.

77. Topol EJ, Ferguson JJ, Weisman HF - Long-term protection from myocardial ischemic events in a randomized trial of brief integrin blockade with percutaneous coronary intervention. JAMA 1997; 278: 479-84.

78. The EPILOG Investigators - Platelet Glycoprotein IIb/IIIa Receptor Blockade and Low-Dose Heparin During Percutaneous Coronary Revascularization. N Engl J Med 1997; 336: 1689-96.

79. The CAPTURE Investigators - Randomised placebo-controlled trial of abciximab before and during coronary intervention in refractory unstable angina:The CAPTURE study. Lancet 1997; 349: 1429-35.

80. White H - Platelet Receptor Inhibition for Ischemic Syndrome Management (PRISM). Presented at the American College of Cardiology. Anaheim, California, USA, 1997.

81. Theroux P - Platelet Receptor Inhibition for Ischemic Syndrome Management in Patients Limited by very Unstable Signs and Symptoms (PRISM-PLUS). Presented at the American College of Cardiology. Anaheim, California, USA, 1997.

82. IMPACT II study group - Randomized placebo-controlled trial of eptifibatide on complications of pecutaneous coronary intervention:IMPACT II. Integrilin to minimize platelet aggregation and coronary thrombosis II. Lancet 1997; 349: 1422-8.

83. The RESTORE investigators - Effects of platelet glycoprotein IIb/IIIa blockade with tirofiban on adverse cardiac events in patients with unstable angina or acute myocardial infarction undergoing coronary angioplasty. Circulation 1997; 96: 1445-53

84. Fischmann DL, Leon MB, Baim DS et al - A randomized comparison of coronarystent placement and balloon angioplasty in the treatment of coronary artery disease. N Engl J Med 1994; 331: 496-501.

85. Serruys PW, De Jaegere P, Kiemeneij Fet al - A comparison of balloon expandable stent implantation with balloon angioplasty in patients with coronary artery disease. N Engl J Med 1994; 331: 489-95.

86. Colombo A, Hall P, Makamura S et al - Intracoronary stenting without anticoagulation accomplished with intravascular ultrasound guidance. Circulation 1995: 91: 1676-88.

87. Serruys PW, Di Mario C - Who was thrombogenic, the stent or the doctor? Circulation 1995; 91: 1891-3.

88. Karillon GJ, Morice MC, Benveniste E et al - Intracoronary stent implantation without ultrasound guidance and with replacement of conventional anticoagulation by anti-platelet therapy:30-day clinical outcome of the French Multicentre Registry. Circulation 1996; 94: 1519-27.

89. Hall P, Nakamura S, Maiello L et al - A ramdomized comparison of combined ticlopidine and aspirin therapy versus aspirin therapy alone after successful intravascular ultrasound-guided stent implantation. Circulation 1996; 93: 215-22.

90. Schomig A, Neumann FJ, Kastrati A et al - A randomized comparison of antiplatelet and anticoagulant therapy after the placement of coronary-artery stents. N Engl J Med 1996; 334: 1084-9.

91. Topol EJ - The stentor and the sea change. J Am Coll Cardiol 1995; 71: 307-8.

92. Rodriguez A, Ambrose JA - Do we require a cure for "stentmania"? J Am Coll Cardiol 1996; 28: 827-9.

93. Serruys PW, Emanuelsson H, van der Giessen W et al - Heparin-coated PalmazSchatz stentz in human coronary arteries: early outcome of the BENESTENT-II Pilot Study. Circulation 1996; 93: 412-22.

94. Roubin GS, Cannon AD, Agrawal SK et al - Intracoronary stenting for acute and threatened closure complicating percutaneous transluminal coronary angioplasty. Circulation 1992; 85: 916-27.

95. Hearn JA, King SB, Douglas JS Jr et al - Clinical and angiographic outcomes after coronary artery stenting for acute or threatened closure after percutaneous transluminal coronary angioplasty. Circulation 1993; 88: 2086-96. 
96. Lincoff AM, Topol EJ, Chapekis AT et al - Intracoronary stenting compared with conventional therapy for abrupt vessel closure complicating coronary angioplasty:a matched case-control study. J Am Coll Cardiol 1993; 21: 866-75.

97. George BS, Voorhees WD 3rd, Roubin GS et al - Multicentre investigation of coronary stenting to treat acute or threatened closure after percutaneous transluminal coronary angioplasty: clinical and angiographic outcomes. J Am Coll Cardiol 1993; 22: 135-43.

98. Serruys PW, Azar AJ, Sigwart U et al - Long-term follow-up of "Stent-like" $(<30 \%$ diameter stenosis post) angioplasty:A case for provisional stenting. J Am Coll Cardiol 1996; 27(suppl): 15A.

99. Piana RN, Moscucci M, Cohen DJ et al - Palmaz-Schatz stenting for treatment of focal vein graft stenosis:immediate results and long-term outcome. J Am Coll Cardiol 1994; 23: 1296-304.

100. Wong SC, Baim DS, Schatz RA et al - Immediate results and late outcomes after stent implantation in saphenous vein graft lesions: The multicenter U.S. PalmazSchatz stent experience. J Am Coll Cardiol 1995; 26: 704-12.

101. Fenton SH, Fischman DL, Savage MD et al - Long-term angiographic and clinical outcome after implantation of balloon-expandable stents in aortocoronary saphenous vein grafts. Am J Cardiol 1994; 74: 1187-91.

102. Thomas M, Hancock J, Holmberg S et al - Coronary stenting following successful angioplasty for total occlusions:preliminary results of a randomized trial. J Am Coll Cardiol 1996; 27(suppl A): 153A.

103. Sirnes PA, Golf S, Myreng Y et al - Stenting in Chronic Coronary Occlusion (SICCO): A randomized controlled trial of adding stent implantation after successful angioplasty. J Am Coll Cardiol 1996; 28: 1444-51.

104. Medina A, Melian F, Suarez de Lezo J, Pan M - Effectiveness of coronary stenting for the treatment of chronic total occlusion in angina pectoris. Am J Cardiol 1994; 73: 1222-4.
105. Goldberg SL, Colombo A, Maiello L et al - Intracoronary stent insertion after balloon angioplasty of chronic total occlusions. J Am Coll Cardiol 1995; 26: 713-9.

106. Erbel R, Haude M, Hopp HW et al - Restenosis Stent(REST) Study:Randomized trial comparing stenting and balloon angioplasty. J Am Coll Cardiol 1996; 27(suppl A): 139A.

107. O'Keefe JH Jr, Hartzler GO, Rutherford BD et al - Left main coronary angioplasty:early and late results of 127 acute and elective procedures. Am J Cardiol 1989; 64: 144-7.

108. Macaya C, Alfonso F, Aniguez A et al - Stenting for elastic recoil during coronary angioplasty of the left main coronary artery. Am J Cardiol 1992; 70 $105-7$.

109. Rechavia E, Litvack F, Mack G, Eigler NL - Stent implantation of saphenous vein graft aorto-ostial lesions in patients with unstable ischemic syndromes:immediate angiorgaphic results and long-term clinical outcome. J Am Coll Cardiol 1995; 24: 866-70.

110. Versaci F, Gaspardone A, Tomai F et al - A comparison of coronary-artery stenting with angioplasty for isolated stenosis of the proximal left anterior descending coronary artery. N Engl J Med 1997; 336: 817-22.

111. Eli Lilly Canada Inc - Data on file.

112. Kereiakes DJ, Lincoff AM, Miller DP, Tcheng JE, Weisman HF, Topol EJ Abciximab reduces risk of unplanned coronary stent deployment: EPILOG trial results. Circulation 1997; 96: I-163.

113. The EPISTENT Investigators. Randomised placebo-controled and balloonangioplasty-controlled trial to assess safety of coronary stenting with use of platelet glycoprotein-IIb/IIIa blockade. Lancet 1998; 252: 87-92.

114. Caramori PRA, German IY, Zago AJ - Reestenose pós-angioplastia. Fisiopatogenia. Arq Bras Cardiol 1997; 69: 141-50. 